\title{
Performance of the Compact High Energy Camera SiPM Prototype Front-End Electronics proposed for the Cherenkov Telescope Array
}

\author{
S A Leach ${ }^{\mathrm{a}, *}$, J S Lapington ${ }^{\mathrm{a}}$, J O D Williams ${ }^{\mathrm{a}}$, C Duffy ${ }^{\mathrm{a}}$, \\ for the CTA GCT project \\ ${ }^{a}$ University of Leicester, $U K$
}

\begin{abstract}
The Compact High Energy Camera (CHEC) is a full-waveform capture camera, designed and proposed for the dual mirror, Schwarzschild-Couder, Small Sized Telescope of the Cherenkov Telescope Array. CHEC-S, the second prototype, is based upon silicon photomultiplier (SiPM) photodetectors optimised for single photon counting and nanosecond timing. The camera liquidcooled focal plane plate comprises a total of 2048 SiPM pixels organised as 32 independent sensor and front-end electronics (FEE) modules providing event detection and signal digitisation of Cherenkov light flashes. Each module comprises an $8 \times 8$ tile of SiPM pixels, coupled to a 64-channel preamplifier-buffer followed by a FEE module based around the TARGET chipset, which combines triggering (T5TEA) and waveform capture (TARGET C) functionality.

We describe a selection of end-to-end performance tests and results conducted at the University of Leicester in the UK. Testing was performed at single module level focusing on characterising the SiPM photodetectors, optimised preamplifier-buffer and updated CHEC-S FEE readout module. Presented results include single photoelectron response, gain-matching, signal range, electronic channel crosstalk, SiPM angular dependency and optical crosstalk.
\end{abstract}

Keywords: CHEC, CTA, front-end electronics, silicon photomultipliers, SST, full-waveform readout

\section{INTRODUCTION}

The CHEC-S camera (Compact High Energy Camera SiPM, Figure 1) has been developed to fulfil the requirement for a compact and economical solution for the small-sized telescope (SST) range of the Cherenkov Telescope Array (CTA) [1]. With over 100 ground-based telescopes, CTA is planned to be the world's largest and most sensitive high-energy gamma-ray observatory. The Southern Hemisphere site in Paranal, Chile, will host 70 SSTs aimed at imaging the Cherenkov light flashes produced during atmospheric interactions of photon energies up to $300 \mathrm{TeV}$.

CHEC-S is the second generation prototype camera (see CHEC-M [2] for the first design) and uses $3 \times 3 \mathrm{~mm}^{2} \mathrm{SiPM}$ photodetectors (Hamamatsu S12642-1616PA pixel array) grouped into fours to create $2048(6 \mathrm{~mm})$ pixels which are optimised for single photon counting and nanosecond timing. The camera focal plane plate is liquid-cooled to maintain a SiPM temperature of $\approx 15^{\circ} \mathrm{C}$ during normal operation. The camera is arranged as 32 identical front-end electronics (FEE) readout modules providing event detection and signal waveform digitisation of Cherenkov light flashes. Each module comprises an $8 \times 8$ arrangement of SiPM pixels, directly mounted to a 64-channel preamplifier-buffer with the signals coupled via 10 $\mathrm{cm}$ coaxial ribbon cables to a CHEC-S FEE module (figure 2). Performance results of the FEE module alone, which is based

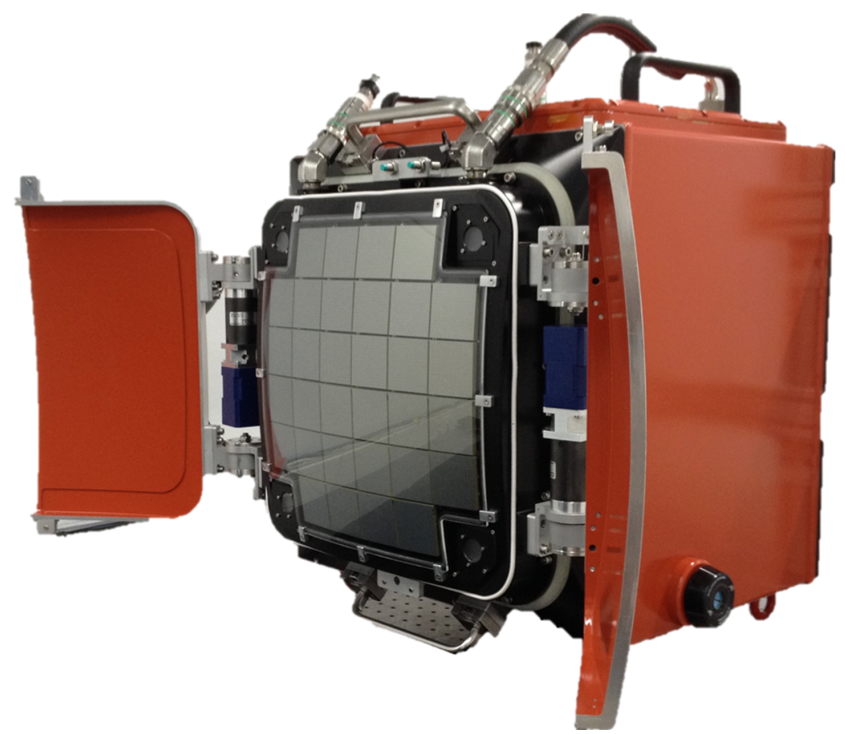

Figure 1: The CHEC-S Camera with 32 photodetector modules, protective window, liquid-cooled focal plane and motorised doors for daytime protection against the harsh desert environment. The detector focal-plane measures $32 \times 32$ $\mathrm{cm}^{2}$ and camera enclosure is approx $53 \mathrm{~cm}$ square. Fully loaded with modules it weighs $47 \mathrm{~kg}$.

\footnotetext{
${ }^{*}$ Corresponding author

Email address: drleach@physicsresearch.co.uk (S A Leach)
} 


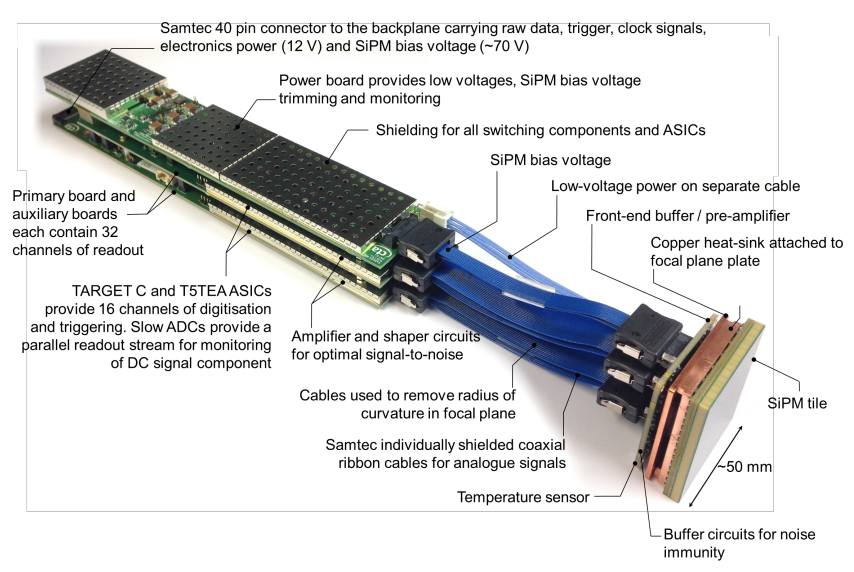

Figure 2: One of 32 identical FEE modules for CHEC-S. Each has 64 independent pixel channels optimised for Cherenkov flashes with full-waveform digitisation of the $8 \mathrm{~ns}$ shaped event pulses (reproduced from [3]).

around the TARGET chipset, have been reported in [3] and we now present end-to-end signal chain results.

\section{FRONT-END ELECTRONICS TESTING}

Detailed characterisation investigations are ongoing at MPI für Kernphysik, Friedrich-Alexander-Universität ErlangenNürnberg, DESY, Nagoya University and at the Space Research Centre, University of Leicester in the UK. The selected results presented here are those obtained at Leicester.

The device under test consists of a single FEE module and SiPM chain. Both are temperature controlled in a similar fashion to CHEC-S using fans and a liquid-cooled mounting block thermally coupled to the SiPM (figure 3). The FEE module consists of a 3 circuit board stack; primary, auxiliary and power supply board (see figure 2 for module summary and [3] for further layout details).

During testing the SiPM can be illuminated with a pulsed laser diode and a diffuse white LED for simulated night sky background enabling many aspects of the end-to-end performance to be investigated. Since CHEC-S uses full-waveform capture, as opposed to peak amplitude only, further information can be gained from each event allowing improved energy extraction of a time-dispersed signal.

\section{SINGLE PHOTOELECTRON DISTRIBUTION}

Each FEE module has 16 independent high voltage (HV) bias channels, supplying groups of 4 pixels known as "Super-Pixels" (SP). This enables control of the SiPM overvoltage (OV) for each SP HV group via an 8-bit digital-to-analogue converter (40 $\mathrm{mV}$ per step). The OV changes the internal gain of each SiPM in the SP and also affects other characteristics including dark count rate, photon detection efficiency (PDE) and optical crosstalk rate. Grouping 4 pixels into SPs reduces the complexity and circuit footprint on the module but does limit the flexibility to set OV for individual SiPMs. However, the $3 \mathrm{~mm}$ SiPMs on each S12642-1616PA array have been matched by

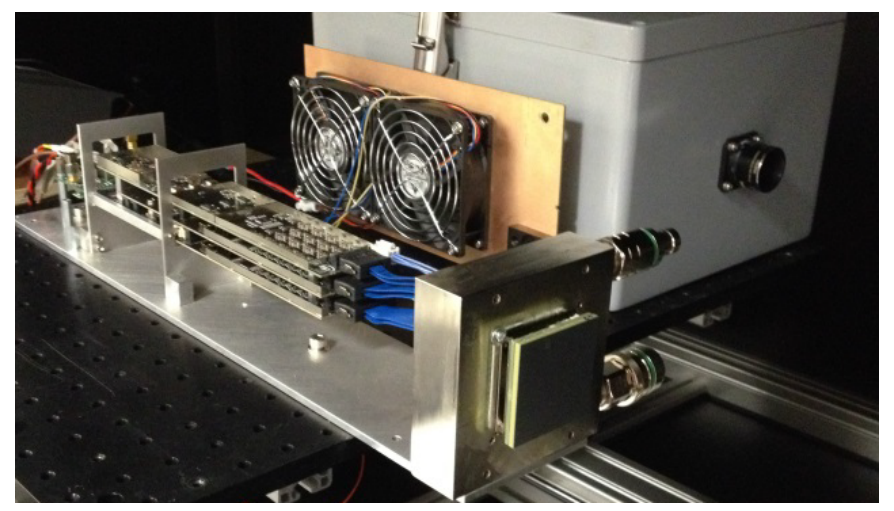

Figure 3: A single CHEC-S FEE module and SiPM chain under test at the Space Research Centre, University of Leicester. The photodetector tile is thermally bonded to the liquid-cooled block to maintain temperature stability.

the manufacturer to ensure a minimal spread in breakdown voltages.

A gain-matching process is used to generate a uniform response from each pixel by employing flat-field illumination of the detector and iteratively adjusting the OV of each SP. Each iterative step involves setting an OV, measuring the amplitude mean from the SP group and adjusting the OV accordingly. Figure 4 illustrates a typical result when illuminating at $\approx 70$ pe (photoelectrons) per pixel. This particular dataset demonstrates a faulty SiPM pixel (23) with another in the SP (pixel 22) compensating for the lower summed signal and illustrates the disadvantage of SP grouping. However, once identified, the effect of faulty and compensating pixels can be calibrated for in camera data.

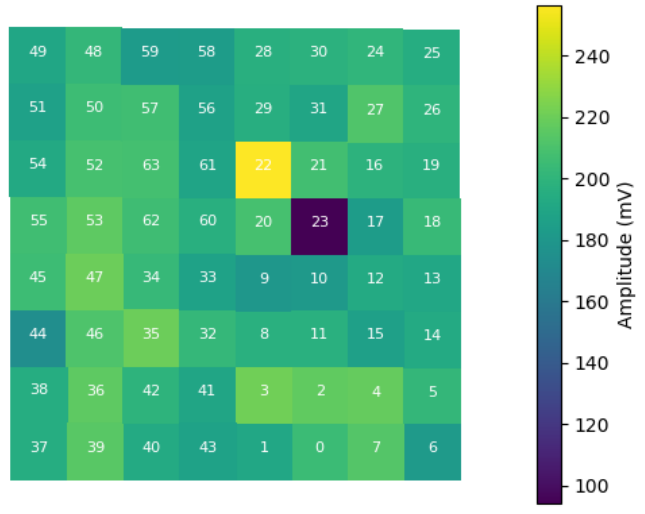

Figure 4: A 64 pixel SiPM response to flat-field illumination $(\approx 70$ pe per pixel) after a gain-matching procedure. This particular image demonstrates a broken pixel 23 and pixel 22 which is compensating for the lower mean amplitude from the SP group.

Figure 5 shows a typical single pe distribution obtained by illuminating a SiPM at low intensity and with an OV of $2.2 \mathrm{~V}$. The histogram reveals a typical Poisson response and allows key performance characteristics to be obtained including signal 


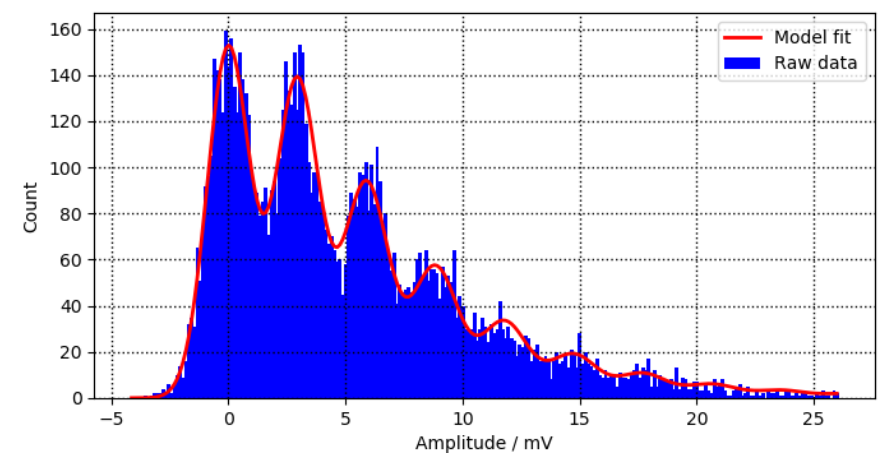

Figure 5: Low intensity single pe distribution showing pedestal and single pe peaks. Operating at $2.2 \mathrm{~V}$ overvoltage the fitting algorithm measures an average pe of 1.3 per laser pulse, signal gain of $2.9 \mathrm{mV} / \mathrm{pe}, 0.9 \mathrm{mV}$ noise and optical crosstalk rate of $30 \%$.

gain, noise, mean pe level and optical crosstalk rate. This single pixel dataset reveals a FEE signal gain of $2.9 \mathrm{mV} / \mathrm{pe}$, noise 0.9 $\mathrm{mV}$, average pe of 1.3 per laser pulse and an optical crosstalk rate of $30 \%$ (inline with the product datasheet). It is worth noting that the S12642 SiPM is an old product and is being superseded with an improved design (increased PDE and lower optical crosstalk rate) for the next iteration of CHEC.

\section{SIGNAL RANGE}

The $3 \mathrm{~mm}$ square SiPMs consist of 3464 micro cells each one $50 \mu \mathrm{m}$ in size and for CHEC-S four SiPMs are connected in parallel to form a single pixel with a capacity to detect over 13,000 photons. Detected photons trigger the SiPM cells producing a signal waveform which is amplified by the preamplifier-buffers. The peak of this waveform is proportional to the total charge and number of photons detected. The waveform shape is then optimised by the analogue FEE and distributed to both the TARGET C digitiser and T5TEA trigger. The input voltage range of the digitiser is $1.9 \mathrm{~V}$. The gain of the SiPM, controlled by the $\mathrm{OV}$, sets the pe dynamic range, above which saturation occurs in the FEE circuit. Figure 6 shows the typical channel response at various OVs before saturation will occur. However, as the CHEC-S FEE digitises and stores the full signal waveform (typically $>100 \mathrm{~ns}$ ), saturated waveform recovery methods can be used to extend the maximum observable pe range well above these levels.

\section{SIPM ANGULAR RESPONSE}

An important requirement for a SST camera is the angular response of the photosensor and readout electronic chain. The Schwarzschild-Couder optical design of the dual-mirror SSTs causes the collected Cherenkov photons to impinge on the surface of the focal plane at angles in the range $30^{\circ}-60^{\circ}$. In order to evaluate the performance of CHEC-S at these angles, the response from a single FEE chain was measured using a diffuse pulsed-laser at varying angles. The pulsed-laser was secured to an optical rail rotated about the SiPM tile in increments of $5^{\circ}$. Figure 7 shows the relative signal peak amplitude from the

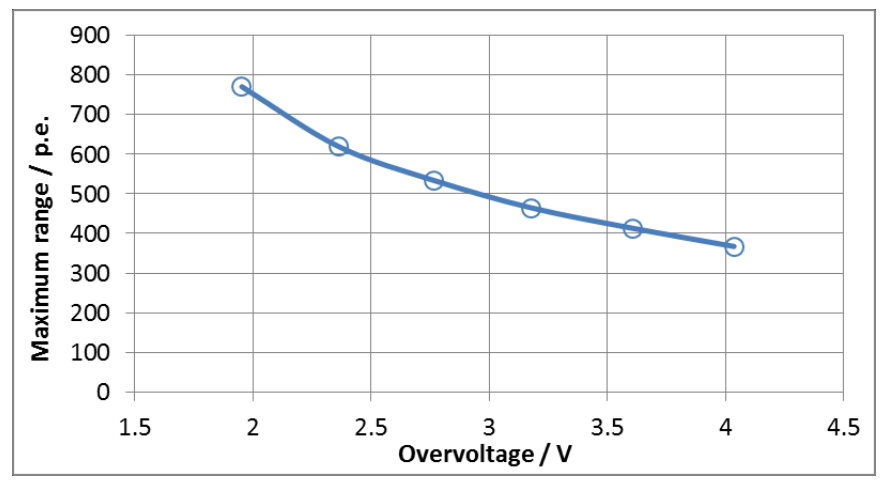

Figure 6: The detectable signal range (prior to FEE signal saturation), in photoelectrons, varies with the SiPM overvoltage and a compromise is made between resolution and range. Using saturated waveform recovery these levels can be extended.

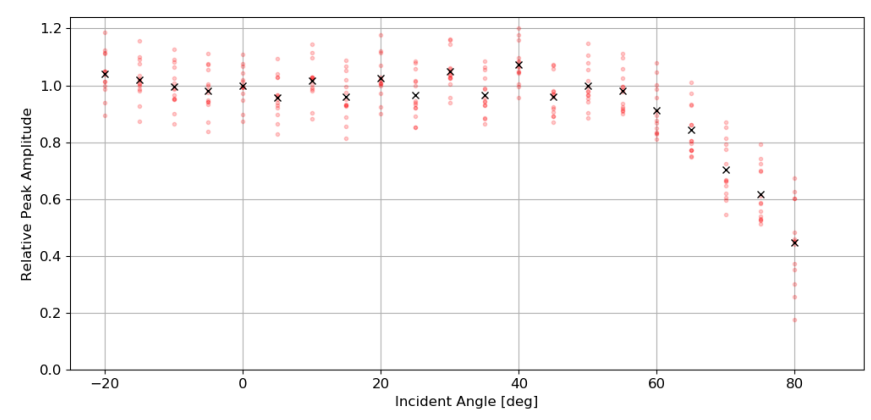

Figure 7: SiPM performance at varying incident angles. Each spread represents the spread in signal peak amplitude from the central column of pixels measured.

SiPM tile as a function of incident angle in the $-20^{\circ}$ to $80^{\circ}$ range. This range was used to show the performance of the photosensor at a high angle of incidence (assuming response symmetry across $\pm 90^{\circ}$ about the normal to the photosensor). The $-20^{\circ}$ to $0^{\circ}$ shows a mirrored response compared to the corresponding positive range, indicating the lack of an offset of the laser at $0^{\circ}$ and hence, a well aligned optical setup.

The vertical spread at each angle shown in Figure 7 represents the spread in signal amplitude across a column of pixels, located along the central vertical line of the SiPM tile, with the mean of these values given by each data point. Central pixels were chosen so the optical distance from pixel to laser would remain unchanged as the laser is rotated about the centre of the SiPM tile. Averaging over the whole tile would provide incomparable data as pixels at the edge of the tile would become closer and further from the laser as it rotated about $0^{\circ}$, hence causing an increase in peak amplitude due to an increase in photon flux for pixels closer to the laser and vice versa.

It can be seen that the angular response of the FEE and photosensor chain is relatively flat and unchanging, up to $55^{\circ}$, before the relative amplitude drops below $95 \%$ of the incident amplitude (at $58^{\circ}$ angle). This indicates that the SiPM tile and FEE module chain implemented in CHEC-S is well suited for Cherenkov imaging cameras using a Schwarzschild-Couder optical design. 


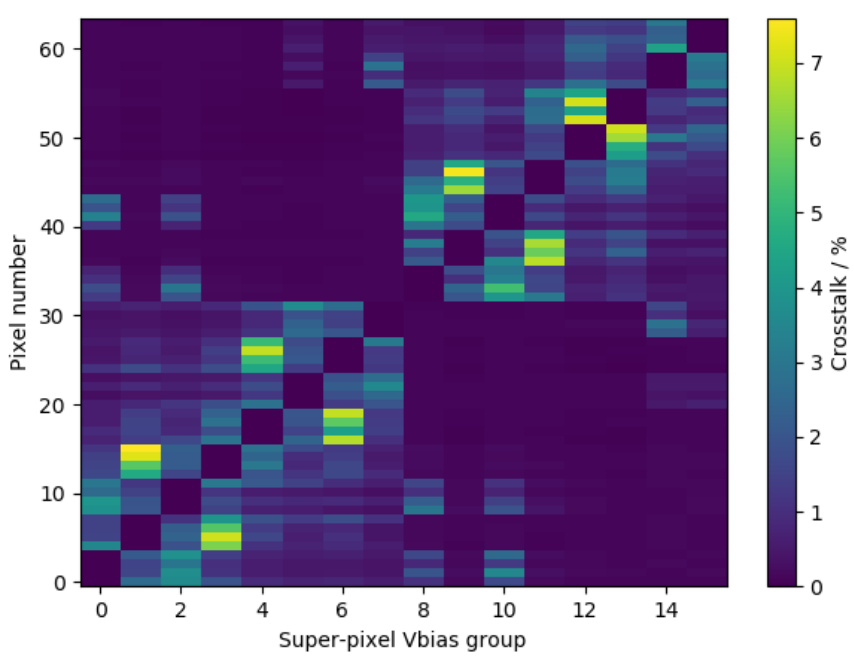

Figure 8: Electronic crosstalk generated per SP (sum of 4 pixels) and measured across the other channels. An improved channel-per-channel measurement is planned.

\section{ELECTRONIC CROSSTALK}

Measuring the electronic crosstalk between channels was achieved using a flat-field illumination, individually activating the HV bias (the OV) for each SP (16 in total) and then comparing the measured signal level with the crosstalk amplitude from the other (non-enabled HV) channels. Figure 8 shows the corresponding crosstalk response for all channels (the ' 0 ' levels are the SPs under test) and indicates some channels with $7 \%$ crosstalk. However, these figures are exaggerated, compared to single channel crosstalk, as this measurement technique activates all 4 pixels in each SP group resulting in an increased crosstalk measurement. An improved channel-perchannel measurement is planned using a focused laser source and illuminating each individual pixel by scanning across the tile. This will help identify where improvements to circuit design and track routing could be implemented.

\section{CONCLUSION}

A second iteration prototype of the FEE for the CHEC camera, a proposed solution designed to meet the requirements for the planned SST class for CTA, has been developed and tested. The FEE modular format reduces manufacturing, commissioning and future maintenance costs as each of the identical 32 units in the camera can be easily replaced as necessary. Endto-end testing of the signal chain demonstrates that the design meets the expected performance for single pe resolution, noise $(0.9 \mathrm{mV})$, detectable signal range $(600$ pe at $2.4 \mathrm{OV})$, photosensor angular response (unchanging below $55^{\circ}$ ) and optical crosstalk rate $(30 \%$ at $2.2 \mathrm{OV})$. Further stand-alone and in camera characterisation of this prototype design is in progress at Leicester and by the other CHEC members.

As planned, several design improvements are being incorporated into a next iteration of the FEE, in particular to accommodate the next generation SiPM (Hamamatsu LVR3) which will substantially reduce optical crosstalk and improve PDE. Other proposed changes include: replacing discrete preamplifier and shaping components with a multi-channel front-end ASIC leading to reduced power consumption; per pixel OV control with a finer resolution to improve gain-matching and allow broken pixels to be isolated; compacting each module onto 2 circuit boards to further reduce costs and improve cooling efficiency; additional temperature sensors for monitoring redundancy and optimising the calibration process; and modifications to signal connectors to improve reliability and ease assembly.

\section{ACKNOWLEDGEMENTS}

We gratefully acknowledge financial support from the agencies and organisations listed here: www.ctaobservatory.org/consortium_acknowledgments

\section{REFERENCES}

[1] M. Actis, et al, Design concepts for the Cherenkov Telescope Array CTA: An advanced facility for ground-based high-energy gammaray astronomy, Exp. Astron 32 (3) (2011) 193-316. doi:10.1007/ s10686-011-9247-0

[2] J. Zorn, et al, Characterisation and testing of CHEC-M - A camera prototype for the small-sized telescopes of the Cherenkov Telescope Array, Nuclear Instruments and Methods in Physics Research Section A: Accelerators, Spectrometers, Detectors and Associated Equipment 904 (2018) 44-63. doi:10.1016/j.nima.2018.06.078

[3] S. Leach, et al, Front-end electronics of the Compact High Energy Camera, Nuclear Instruments and Methods in Physics Research Section A: Accelerators, Spectrometers, Detectors and Associated Equipment. (2019) doi : 10.1016/j.nima.2018.12.061 